\title{
Inhibitory effect of polyunsaturated fatty acids on the growth of Helicobacter pylori: a possible explanation of the effect of diet on peptic ulceration
}

\author{
L Thompson, A Cockayne, R C Spiller
}

\begin{abstract}
Diets high in polyunsaturated fatty acids may protect against duodenal ulcer, possibly through inhibiting the growth of Helicobacter pylori. This hypothesis was tested in vitro by incubating $H$ pylori microaerophilically with a range of polyunsaturated fatty acids. $\omega-3$ Linolenic acid significantly, but reversibly, inhibited growth at $1.8,2.5$, and $5 \times 10^{-4} \mathrm{M}$ $(\mathbf{p}<0 \cdot 01)$, while concentrations of $10^{-3} \mathrm{M}$ killed virtually all organisms, with cell lysis observed by electron microscopy. Similar inhibitory effects were seen with other polyunsaturated fatty acids, at concentrations of $2.5 \times 10^{-4} M$ the relative inhibitory potencies were oleic (C18:1) < linoleic (C18:2)< arachidonic $(C 20: 4)<\omega-3$ linolenic $(C 18: 3)=\omega-6$ linolenic $(\mathrm{C} 18: 3)=$ eicosapentanoic $(\mathrm{C} 20: 5)$ acid. Cell fractionation studies with ${ }^{14} \mathrm{C}$ labelled linolenic acid showed that the linolenic acid was associated with the membrane fraction. Commonly ingested dietary polyunsaturated fatty acids inhibit the growth of $H$ pylori in vitro, an effect which deserves further in vivo study. (Gut 1994; 35: 1557-1561)
\end{abstract}

The scientific revolution which has accompanied the recognition of the importance of Helicobacter pylori, has left in the shadows a body of information relating to possible dietary influences on the incidence of peptic ulceration. ${ }^{1-4}$ Wide variations in the incidence of peptic ulcer in different African populations can be related to the apparent protective effect of high intakes of fresh fruit and vegetables, while diets high in refined starch seemed detrimental. ${ }^{4}$ Interest in the role of polyunsaturated fatty acids was stimulated considerably by Hollander and Tarnawski, ${ }^{5}$ who linked the decline in duodenal ulcer to the rise in dietary consumption of polyunsaturated fatty acids. The subsequent finding that ulcer patients had a lower intake of linoleic acid further supported the idea. ${ }^{6}$ These authors have linked the protective effect of polyunsaturated fatty acids to the synthesis of mucosal prostaglandins which may indeed be important in some of the experimental models of ulceration. ${ }^{7}$
Other mechanisms are possible, however, and we were interested in the possibility, long recognised by veterinarians, that long chain fatty acids inhibit gastric bacterial metabolism. ${ }^{8-11}$ We have confirmed these effects in human colonic bacteria, showing that increasing unsaturation in the $\mathrm{C} 18$ series leads to increasing antibacterial potency. ${ }^{12}$ These effects, unlike other reports, ${ }^{13}$ do not depend on lipid peroxidation as they are observed in an anaerobic environment.

The evident importance of $H$ pylori infection in human peptic ulcer together with the above mentioned antibacterial effects of fatty acids, caused us to explore the hypothesis that dietary polyunsaturated fatty acids directly inhibit the intragastric growth of $H$ pylori. We therefore performed a detailed, in vitro, dose-response study of inhibition of the growth of $H$ pylori by two of the commonest dietary polyunsaturated fatty acids - $\omega-3$ linolenic acid (C18:3) and linoleic acid (C18:2) - at a range of concentrations likely to be found postprandially in the human stomach. We also examined reversibility of the effect and looked at the intracellular distribution of ${ }^{14} \mathrm{C}$ labelled linolenic acid. Finally, we investigated the potency of a wider range of polyunsaturated fatty acids and showed that this inhibitory effect is a general property of these substances.

\section{Methods}

$H$ pylori (NCTC 11637) was supplied by the National Collection of Type Cultures, Colindale, England. Before these experiments the organism had been stored at $-70^{\circ} \mathrm{C}$ in fetal calf serum (FCS). For the duration of the study the bacteria were routinely cultured every 72 hours onto chocolate agar plates (CHP). The plates were incubated at $37^{\circ} \mathrm{C}$ under microaerophilic conditions $(6 \%$ oxygen). Throughout the study $H$ pylori broth cultures were incubated microaerophilically at $37^{\circ} \mathrm{C}$ in a shaking $(150 \mathrm{rpm})$ incubator, using Isosensitest Broth (ISB) (Oxoid, Basingstoke, England) containing 5\% FCS (Gibco BRL, Paisley, Scotland) in addition to the test substances identified below. The fatty acids were all purchased from the Sigma Chemical Co, Poole, Dorset. The purity was approx $99 \%$ in all cases except for $\omega-3$ linolenic

Department of
Therapeutics, L Thompson 11 March 1994
} 
acid and arachidonic which had purities of approximately $98 \%$ and $90 \%$ respectively.

Preliminary screening studies were performed using flood plates of confluent growth on which were laid $8 \mathrm{~mm}$ discs of filter paper soaked with $10 \mu \mathrm{l}$ of $0,0 \cdot 1,1$, and $10 \%$ $\mathrm{mg} / \mathrm{ml}$ long chain fatty acids dissolved in ethanol. While $\omega-3$ linoleic acid showed a ring of inhibition, even at a concentration of $0.1 \%$ $\mathrm{mg} / \mathrm{ml}$, none of the saturated fatty acids tested (palmitic (C16:0), stearic (C18:0) or arachidic (C20:0)) showed any inhibitory zone. We therefore proceeded to examine only the unsaturated fatty acids in subsequent studies.

Growth of $H$ pylori was assessed from the change in the optical density (OD) measured at $540 \mathrm{~nm}$ using a Shimadzu UV-160A spectrophotometer (V A Howe \& Co Ltd, Oxon, England).

\section{STUDY 1}

\section{Effect of $\omega-3$ linolenic acid (C18:3) on bacterial} growth

$H$ pylori was grown for 24 hours in ISB yielding a viable count of $1 \times 10^{8} \mathrm{cfu} / \mathrm{ml}$. It was then diluted 1:5 with fresh ISB to give an initial OD $(540 \mathrm{~nm})$ of 0.1 and an initial volume of $1 \mathrm{ml}$. Incubations were performed in 24 well tissue culture clusters (Costar, High Wycombe, Bucks). A range of $\omega-3$ linolenic acid solutions diluted in ethanol was added to the wells giving a fatty acid concentration of between $1 \mathrm{mM}$ and $0.01 \mathrm{mM}$. Each experiment consisted of six control wells to which $11.2 \mu l$ of ethanol had been added and six test wells to which $11.2 \mu$ l of the appropriate fatty acid/ethanol solution had been added. Cultures were incubated for 24 hours and bacterial growth was assessed at time 0 and time +24 hours.

\section{Motility assessment}

The effect of the various fatty acid concentrations on bacterial motility was determined using phase contrast microscopy $(\times 400)$. Motility was assessed using a semiquantitative scoring system as follows: $0=$ no motile rods seen; $1=1-5$ motile rods visible per high power field (HPF); $2=>20$ rods per $\mathrm{HPF}<50 \%$ rods motile; $3=>20$ rods per $\mathrm{HPF},>50 \%$ rods motile.

\section{Recovery from inhibition}

The ability of the organism to recover after exposure to $\omega-3$ linolenic acid was studied by inoculating $0.9 \mathrm{ml}$ fresh ISB with $0.1 \mathrm{ml}$ of fatty acid-exposed cells. Once again each experiment comprised different concentrations of fatty acid-exposed cells, which were tested in groups of six. These were compared with six control wells where the inoculum had not been exposed to $\omega-3$ linolenic acid. Broths were incubated for 24 hours as previously described and the ability to recover was assessed by measuring the $O D(540 \mathrm{~nm})$ at time 0 and at time +24 hours.
The effect of linoleic acid (C18:2) on bacterial growth, over the same concentration range, was also studied, as was the ability of the organisms to recover after exposure to C18:2.

\section{STUDY 2}

\section{Incorporation of ${ }^{14} C+\omega-3$ linolenic acid into} $\mathrm{H}$ pylori

A large amount of organism was prepared by inoculating $12 \times 7.5 \mathrm{ml}$ ISB with $200 \mu \mathrm{l}$ of a broth culture containing $>50 \%$ motile rods. After incubation for 48 hours, the culture was pelleted by centrifugation at $4000 \mathrm{~g}$ for 10 minutes. The pellet was resuspended in $22 \mathrm{ml}$ of ISB and then split into $2 \times 11 \mathrm{ml}$ samples with an initial OD (540 nm) of 1.687 and a viable count of $7 \times 10^{8} \mathrm{cfu} / \mathrm{ml}$. Some $12 \cdot 5$ $\mu \mathrm{l}(1 \cdot 25 \mu \mathrm{Ci}){ }^{14} \mathrm{C}$-linolenic acid (Amersham International plc, Little Chalfont, Bucks) were added to one of the samples. Both suspensions were then incubated in air at $37^{\circ} \mathrm{C}$ with shaking $(150 \mathrm{rpm})$ for one hour. Suspensions were centrifuged at $4000 \mathrm{~g}$ for 10 minutes, and the resulting pellets were washed twice with $5 \mathrm{ml}$ phosphate buffered saline (PBS). Pellets were resuspended in $5 \mathrm{ml}$ fresh PBS, cooled, and sonicated until the cells had lysed. This was checked by examining the solutions under phase contrast $(\times 400)$. Membranes were pelleted by centrifugation at $100000 \mathrm{~g}$ for one hour. Supernatants were retained for scintillation counting. Pellets were washed with $10 \mathrm{ml}$ PBS at $100000 \mathrm{~g}$ for 30 minutes and resuspended, with gentle sonication, in $3 \mathrm{ml}$ sterile distilled water. Radioactivity was measured by adding a $250 \mu$ l aliquot of either the control supernatant or pellet suspension, or the radiolabelled supernatant or pellet suspension to $3 \mathrm{ml}{ }^{14} \mathrm{C}$ scintillant ( $3 \mathrm{~g} \mathrm{PPO}$ (2,5 diphenyloxazole) and $0 \cdot 1 \mathrm{~g}$ POPOP (1,4-di $\{2,5$ diphenyloxazoyl $\})$ both supplied by Koch-light Ltd, Hatfield, Herts, in 11 low sulphur grade toluene. Duplicate samples were counted for 10 minutes (error $=1 \%$ ) using a Kontron Intertechnique SL 3000 scintillation counter (Kontron, St Albans, Hertfordshire).

\section{STUDY 3}

\section{Effect of a range of long chain fatty acids}

(LCFAs) on bacterial growth

$H$ pylori was grown as described above but this time the effect of a range of LCFAs on bacterial growth was studied. The LCFAs investigated, at a concentration of $0.25 \mathrm{mM}$, were oleic acid (C18:1), linoleic acid (C18:2), linolenic acid (C18:3), both $\omega-3$ and $\omega-6$ isomers, arachidonic acid (C20:4), and eicosapentaenoic acid (EPA) (C20:5). Growth was assessed from the mean (SEM) change in OD (540 nm) during the 24 hours' incubation period $(n=6)$. Additional experiments were performed with thymol-free catalase (Sigma Chemical Co Ltd), Poole, Dorset) added to the culture at a concentration of $10 \mu \mathrm{g} / \mathrm{ml}$, previously shown to protect against peroxidation products. ${ }^{13}$ 


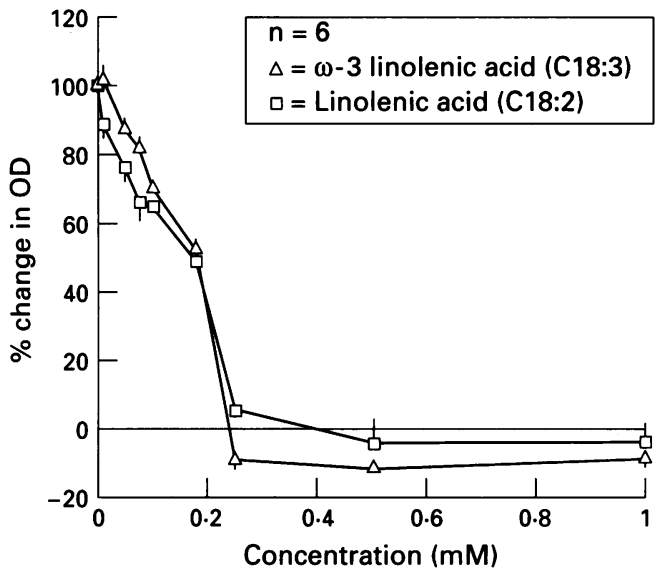

Figure 1: Effect of $\omega-3$ linolenic acid (C18:3) and linolenic acid (C18:2) on the growth of Helicobacter pylori. Growth is expressed as the percentage rise in optical density (OD) expressed as a percentage of control, and showed significant $(p<0.01)$ inhibition at all concentrations used, except $0.01 \mathrm{mM}$ of both fatty acids.

\section{STUDY 4}

Comparison of effect of $\omega-3$ linoleic acid and trilinolenin

$H$ pylori was incubated as before with either free fatty acid or the neutral triglyceride, trilinolenin (C18:3), at concentrations of 1 , $0.5,0 \cdot 1,0.05$, and $0.01 \mathrm{mM}$ for 17 hours.

CALCULATIONS AND STATISTICS

Results are expressed in the test as the mean (SEM). Statistical significance was determined using the Mann-Whitney U test.

\section{Results}

STUDY 1

Effect of $\omega-3$ linolenic acid on growth of

$\mathrm{H}$ pylori

Under the control experimental conditions, $H$ pylori grew steadily reaching a maximum viable count at about $18-24$ hours of approximately $2 \times 10^{8}$ organisms per ml. Both $\omega-3$ linolenic acid (C18:3) and linoleic acid (C18:2) significantly inhibited the growth of $H$ pylori at concentrations above $0 \cdot 1 \mathrm{mM}$, with effectively no growth above $0.18 \mathrm{mM}, \mathrm{p}<0.01$ (Fig 1). The concentration required to inhibit growth by $50 \%$, the inhibitory dose 50 (ID50) was $0.2 \mathrm{mM}$ for both fatty acids. Motility

Effect of $\omega-3$ linolenic acid on appearance and motility of Helicobacter pylori

\begin{tabular}{|c|c|c|c|c|}
\hline \multirow{3}{*}{$\begin{array}{l}\text { Concentration } \\
\text { of } \omega-3 \\
\text { linolenic acid } \\
(m M)\end{array}$} & \multicolumn{4}{|l|}{ Time } \\
\hline & \multicolumn{2}{|l|}{ O Hours } & \multicolumn{2}{|l|}{24 Hours } \\
\hline & Motility ${ }^{\star}$ & Appearance & Motility* & Appearance \\
\hline Control & 3 & Spiral rods & 3 & Spiral rods \\
\hline & 3 & Spiral rods & 3 & Spiral rods \\
\hline 0.05 & 3 & Spiral rods & 3 & Spiral rods \\
\hline 0.08 & 3 & Spiral rods & 3 & Spiral rods \\
\hline $0 \cdot 1$ & 2 & Spiral rods & 2 & Spiral rods \\
\hline $0 \cdot 18$ & 2 & Spiral rods & $\overrightarrow{1}$ & Uneven surface \\
\hline 0.25 & 1 & Ûneven surface & 0 & Elongated rods \\
\hline 0.5 & 0 & Uneven surface & 0 & Cell lysis \\
\hline
\end{tabular}

${ }^{\star} 0=$ no motile rods seen, $1=1-5$ motile rods visible per high power field (HPF), $2=>20$ rods per $\mathrm{HPF},<50 \%$ rods motile, $3=>20$ rods per $\mathrm{HPF},>50 \%$ rods motile.

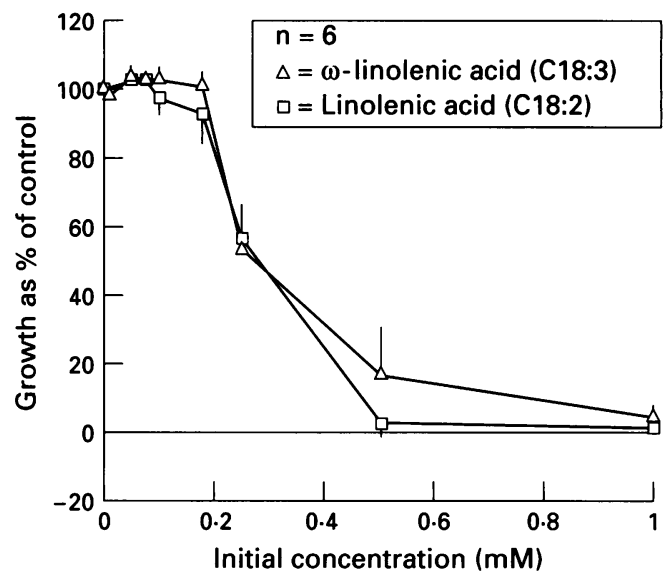

Figure 2: Recovery from exposure to polyunsaturated fatty acids. After initial incubation at the concentrations shown, organisms were incubated in a fatty acid-free medium and growth assessed from the rise in optical density expressed as a percentage of control. Recovery was virtually complete at the lower concentrations but significantly reduced $(p<0 \cdot 01)$ for all concentrations above $0.05 \mathrm{mM}$, with no growth after exposure to 0.5 and $1 \mathrm{mM}$ polyunsaturated fatty acid.

studies (Table) showed similar inhibition of motility, which was obvious at concentrations of $0.1 \mathrm{mM}$, increased with higher concentrations and was virtually complete at $0.25 \mathrm{mM}$ or above. Phase contrast microscopy also showed morphological changes at the higher concentrations $(>0.18 \mathrm{mM})$, and electron microscopy showed abnormal cell forms with outer membrane blebbing, surface irregularities, and cell lysis at $10^{-3} \mathrm{M}$. Resuspending cells in fatty acid-free medium confirmed that no viable cells were found after incubation with $1 \mathrm{mM}$. With concentrations $<0 \cdot 18 \mathrm{M}$ complete recovery was seen, with final counts comparable to control values, however. Incomplete recovery was seen with intermediate concentrations (Fig 2).

\section{STUDY 2}

Incorporation of ${ }^{14} \mathrm{C} \omega-3$ linolenic acid into $\mathrm{H}$ pylori

Most $(82 \%)$ of the $H$ pylori associated radiolabel appeared in the membrane pellet, with only $18 \%$ in the supernatant, implying that during the one hour culture the fatty acid had become associated with, or incorporated into, the bacterial outer membrane.

\section{STUDY 3}

Effect of a range of LCFAs on bacterial growth Using a concentration $(0.25 \mathrm{mM})$ near to the ID50 of both linoleic and $\omega-3$ linolenic acid, clear inhibition of growth was shown for all polyunsaturated fatty acids studied (Fig 3). The higher degree of unsaturation gave slightly greater inhibition but even oleic acid still had a highly significant effect.

Addition of catalase alone to the control broths did not significantly alter growth, which was $88.2(2 \cdot 2) \%$ of control; nor did it alter the effect of $0.25 \mathrm{mM} \omega-3$ linolenic acid - growth was $3.0(0.8) \%$, which was not 


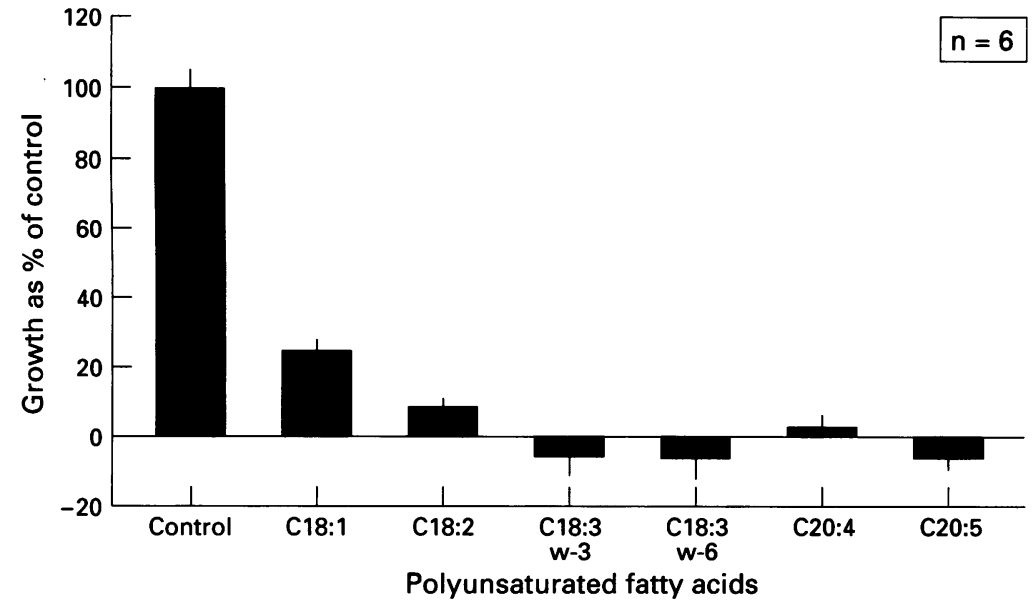

Figure 3: Effect of a range of polyunsaturated fatty acids on the growth of $\mathbf{H}$ pylori. Growth was significantly inhibited $(p<0.01)$ by all polyunsaturated fatty acids tested. There was, however, a significantly greater effect with the increasing degree of unsaturation. Both $\omega-3$ linolenic acid (C18:3) and $\omega-6$ linolenic acid (C18:3) were significantly more inhibitory than $C 18: 2$, which was in turn significantly more inhibitory than C18:1 (all comparisons $p<0 \cdot 01, n=6$ ).

significantly different from the control value of $5 \cdot 0(0 \cdot 8) \%$.

\section{STUDY 4}

Comparison of effect of $\omega-3$ linolenic acid and trilinolenin

The neutral triglyceride, trilinolenin, exerted a much reduced inhibitory effect compared with $\omega-3$ linolenic acid. Growth was significantly reduced only at concentrations of $1 \mathrm{mM}$ trilinolenin, while the free acid was inhibitory at concentrations as low as $0.05 \mathrm{mM}$ (Fig 4).

\section{Discussion}

Our study confirms the inhibitory effect in vitro of polyunsaturated fatty acids on the growth of $H$ pylori. Other studies in anaerobic environments $^{910}$ have shown similar inhibition, which has been thought to be due to damage to the bacterial outer lipid

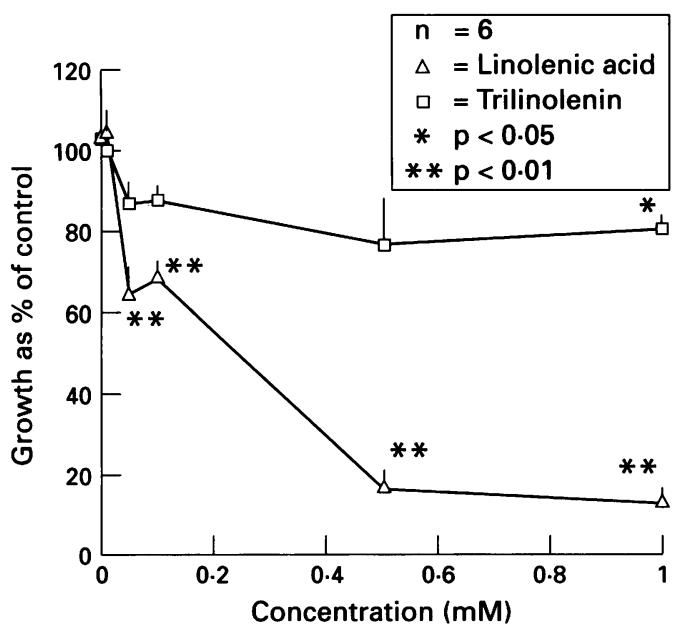

Figure 4: Comparison of the effects of trilinolenin and linoleic acid on the growth of $\mathrm{H}$ pylori. The free acid was more inhibitory than the triglyceride, showing significant inhibition compared with control values at all concentrations $>0.01 \mathrm{mM}(p<0.01, n=6)$, whereas the triglyceride showed significant inhibition only at $1 \mathrm{mM}$ $(p<0 \cdot 05, n=6)$. membrane. ${ }^{14}$ Our electron micrographs confirm that at high concentrations polyunsaturated fatty acids disrupt the cell membrane leading to cell lysis. We also found that at lower concentrations, before irreversible cell damage has occurred, motility is significantly inhibited after 24 hour incubations. Polyunsaturated fatty acids increase membrane fluidity, and hence permeability to small molecules such as water, ${ }^{15}$ chloride, ${ }^{16}$ and drugs. ${ }^{17}$ When polyunsaturated fatty acids are added to aerobic cultures of Gram positive organisms, amino acid uptake is impaired and leakage of labelled amino acids out of the cell is increased while oxygen consumption is stimulated, a combination which has been suggested to reflect increased permeability of mitochondrial and other cell membranes to protons. ${ }^{18}$

Others have suggested that when organisms are incubated aerobically with arachidonic acid, they are killed by toxic lipid peroxides ${ }^{13}$ and that this can be inhibited by catalase. We have described similar antibacterial effects to those described above in an anaerobic environment ${ }^{12}$ and believe that under these conditions other mechanisms must be invoked. Polyunsaturated fatty acids are readily incorporated into the outer cell membranes of Gram negative organisms, where they significantly increase membrane fluidity. It is possible that by opening up permeability channels the concentration gradients necessary between the organism and its environment, such as those for hydrogen ions, may be dissipated, with fatal results. The lack of effect of saturated fats may be due to their reduced solubility and fluidity which makes it less likely that they will readily be incorporated into bacterial membranes.

The concentrations of polyunsaturated fatty acids we have studied would frequently be obtained in the human stomach, in which the daily intake of $1-20 \mathrm{~g} /$ day $^{19}$ is diluted in $2-4$ litres, giving a mean concentration ranging from 1-35 mM. Obviously, after eating fatty foods the concentration will be much higher before dilution with gastric secretion and ingested fluids. Free fatty acids are foul tasting and are not generally ingested but are taken as neutral triglycerides, which as our study 4 showed, are much less potent antibacterial agents. The gastric fundus, however, contains substantial amounts of acid stable lipase activity, equal to $20 \%$ of the total pancreatic lipase. $^{20}$ As a consequence, dietary neutral lipid is approximately $10 \%$ hydrolysed intragastrically ${ }^{21}$ and concentrations of free fatty acids can be predicted to be approximately a tenth of total lipid, still well within the concentration $(>0.05 \mathrm{mM})$ we have shown to be bacteriocidal. It is, however, worth noting that $H$ pylori lies beneath a protective mucus layer which may well reduce the free fatty acid concentration still further. Direct measurement of the concentration beneath the undisturbed mucus layer would be very difficult and it may be that the only way to address this point will be to perform a clinical trial of the effect of polyunsaturated fatty acid 
supplementation on $H$ pylori infection, a study which we are currently undertaking.

Much interest was generated by Hollander and Tarnawski when they suggested that the decline in duodenal ulcer incidence from 1940 onwards was related to appreciable increases in the dietary intake of polyunsaturated fatty acids. ${ }^{5}$ At that time emphasis was on the possible influence on prostaglandin metabolism, which subsequent studies supported, showing that linoleic acid emulsion increased, at least temporarily, the gastric secretion of prostaglandin E. ${ }^{22}$ Epidemiological studies in both Edinburgh ${ }^{6}$ and Dublin ${ }^{23}$ have shown that patients with duodenal ulcers have reduced adipose tissue linoleate levels, a measure which has been shown to correlate well with overall dietary intake. ${ }^{24}$ These associations are difficult to interpret, however, since dietary intake increases with increasing socioeconomic status $^{23}$ and declines with smoking. 6192526 Smoking seems to inhibit both polyunsaturated fatty acid and fibre intake, and its effect is much stronger than social class. ${ }^{19}$ The relationship between linoleic acid intake and ulcers seems to depend heavily on the effect of smoking, since if one excludes heavy smokers, the difference between ulcer patients and controls becomes insignificant. ${ }^{27}$ Smokers' diets differ in other important respects, including high salt and low fibre intake, ${ }^{19}$ so caution is needed before laying responsibility at the door of any particular dietary component.

Thus, there are many potentially beneficial effects of dietary lipid on the stomach, including indirect effects on mucosal prostaglandins, ${ }^{7}$ direct effects on mucosal hydrophobicity, ${ }^{28}$ and now, as we have shown, direct effects on $H$ pylori and its growth and production of toxins. Plainly, the picture is evolving and becoming more sophisticated and further research is needed before we can answer our ulcer patient's frequently asked, and seemingly simple question, 'does my diet matter?'

These data were presented in part at the Vth Workshop on Gastroduodenal Pathology and Helicobacter pylori held at Trinity College, Dublin July 1992 . This study was supported by Trent Regional Health Authority.

1 Malhotra SL. Peptic ulcer in India and its aetiology. Gut 1964; 5: 412-15.

2 Malhotra SL. A comparison of unrefined wheat and rice diets in the management of duodenal ulcer. Postgrad Med f $1978 ; 54: 6-9$.

3 Tovey FI, Tunstall $M$. Duodenal ulcer in black populations in Africa south of the Sahara. Gut 1975; 16: 564-76.

4 Rydning A, Aadland E, Berstad A, Odegaard B. Prophylactic effect of dietary fibre in duodenal ulcer disease. Lancet 1982; ii: 736-7.
5 Hollander D, Tarnawski A. Dietary essential fatty acids and decline in peptic ulcer disease - a hypothesis. Gut 1986; 27: $239-42$

6 Grant HW, Palmer KR, Riermesma RR, Oliver MF. Duodenal ulcer is associated with low dietary linoleic acid intake. Gut 1990; 31: 997-8.

7 Tarnawski A, Hollander D, Gergely H. Protection of the gastric mucosa by linoleic acid - a nutrient essential fatty acid. Clin Invest Med 1987; 10: 132-5.

8 Czerkawski JW, Blaxter KL, Wainman FW. The metabolism of oleic, linoleic and linolenic acids by sheep with reference to their effects on methane production. $B r f$ Nutr 1966; 20: 349-61.

9 Henderson C. The effects of fatty acids on pure cultures of rumen bacteria. Fournal of Agricultural Science 1973; 81: 107-12.

10 Maczulak AE, Dehority BA, Palmqvist DL. Effects of longchain fatty acids on growth of rumen bacteria. Appl Environ Microbiol 1981; 42: 856-62.

11 Chalupa W, Rickabaugh B, Kronfield DS, Sklan D. Rumen fermentation in vitro as influenced by long chain fatty acids. F Dairy Sci 1984; 67: 1439-44.

12 Thompson L, Edwards R, Greenwood D, Spiller RC. Possible mechanisms of alterations in colonic flora by diets high in polyunsaturated fatty acids. In: Duerden BI, Brazier JS, Seddon SV, Wade WG, Eds. Medical and environmental aspects of anaerobes. Petersfield: Wrightson Biomedical Publishing Ltd, 1992: 144-8.

13 Knapp HR, Melly A. Bactericidal effects of polyunsaturated fatty acids. F Infect Dis 1986; 154: 84-94.

14 Galbraith H, Miller TB. Physicochemical effects of long chain fatty acids on bacterial cells and their protoplasts. f Appl Bacteriol 1973; 36: 647-58.

15 Hagve TA. Effects of unsaturated fatty acids on cell membrane functions. Scand $\mathcal{f}$ Clin Lab Invest 1988; 48: 381-8.

16 Schaeffer BE, Zadunaisky JA. Stimulation of chloride transport by fatty acids in corneal epithelium and relation to changes in membrane fluidity. Biochim Biophys Act 1979; 556: 131-43.

17 Hori R, Kagimoto Y, Kamiya K, Inui KI. Effects of free fatty acids as membrane components on permeability of drugs across bilayer lipid membranes. Biochim Biophys Act drugs across bilayer

18 Galbraith $H$, Miller TB. Effect of long chain fatty acids on bacterial respiration and amino acid uptake. $\mathcal{f} A p p l$ Bacteriol 1973; 36: 659-75.

19 Fulton M, Thomson M, Elton RA, Brown S, Wood DA Oliver MF. Cigarette smoking, social class and nutrient intake: relevance to coronary heart disease. Eur f Clin Nutr 1988; 42: 797-803.

20 Moreau H, Laugier R, Gargouri Y, Ferrato F, Verger R. Human preduodenal lipase is entirely of gastric fundic origin. Gastroenterology 1988; 95: 1221-6.

21 Borgstrom B, Dalhqvist A, Lundh G, Sjovall J. Studies of intestinal digestion and absorption in the human. $f$ Clin invest 1957; 36: 1521-36.

22 Grant HW, Palmer KR, Kellt RW, Wilson NH, Misiewicz J. Dietary linoleic acid, gastric acid, and prostaglandin JJ. Dietary linoleic acid, gastric acid, and pros
secretion. Gastroenterology 1988; 94: 955-9.

23 Kearney J, Kennedy NP, Keeling PWN, Keating JJ, Grubb L, Kennedy M, Gibney MJ. Dietary intakes and adipose tissue levels of linoleic acid in peptic ulcer disease. Br F Nutr 1989; 62: 699-706.

24 van Staveren WA, Deurenberg P, Katan MB, Burema J, De Groot LCPGM, Hoffmans MDAF. Validity of the fatty acid composition of subcutaneous fat tissue microbiopsies as an estimate of the long-term average fatty acid composition of the diet of separate individuals. Am 7 Epidemiol 1986; 123: 455-63.

25 Wood DA, Butler S, Macintyre C, Riemersma RA, Thomson M, Elton RA. Linoleic and eicosapentaenoic acids in adipose tissue and platelets and risk of coronary acids in adipose tissue and platelets a
heart disease. Lancet 1987; i: 177-81.

26 Oliver MF. Cigarette smoking, polyunsaturated fats, linoleic acid, and coronary heart disease. Lancet 1989; ii: 1241-3.

27 Seidelin KN, Meisner S, Bukhave K. Percentage distribution of fatty acids in subcutaneous adipose tissue of patients with peptic ulcer disease. Am $\mathscr{f}$ Clin Nutr 1993; 57: 70-2.

28 Dial EJ, Lichtenberger LM. Milk protection against experimental ulcerogenesis in rats. Dig Dis Sci 1987; 32: 1145-50. 